\title{
Quantum Monte Carlo Calculations of Dihydrogen Binding Energetics on Ca Cations: an Assessment of Errors in Density Functionals for Weakly Bonded Systems
}

\author{
Michal Bajdich, ${ }^{1}$ Fernando A. Reboredo, ${ }^{1}$ and P. R. C. Kent ${ }^{2}$ \\ ${ }^{1}$ Materials Science and Technology Division, Oak Ridge National Laboratory, Oak Ridge, TN 37831, USA \\ ${ }^{2}$ Center for Nanophase Materials Sciences, Oak Ridge National Laboratory, Oak Ridge, TN 37831, USA
}

(Dated: October 30, 2018)

\begin{abstract}
We investigate the binding of single and quadruple hydrogen molecules on a positively charged $\mathrm{Ca}$ ion. By comparing with benchmark quantum Monte Carlo (QMC) calculations we demonstrate wide variability in other more approximate electronic structure methods including common density functionals. Single determinant QMC calculations find no binding at short range by approximately $0.1 \mathrm{eV}$ for the quadruple hydrogen molecule case, for a fixed hydrogen bond length of 0.77 Angstrom. Density functional calculations using common functionals such a LDA and B3LYP differ substantially from the QMC binding curve. We show that use of full Hartree-Fock exchange and PBE correlation (HFX+PBEC) obtains close agreement with the QMC results, both qualitatively and quantitatively. These results both motivate the use and development of improved functionals and indicate that caution is required applying electronic structure methods to weakly bound systems such as hydrogen storage materials based on metal ion decorated nanostructures.
\end{abstract}

PACS numbers:

Our ability to accurately predict molecular adsorption energies is of widespread importance in the physical, chemical, and materials sciences. Technologically, the adsorption of small molecules on semiconductors or metals is an essential step in many catalytic or energy storage related areas. In the case of hydrogen storage, the strength of the adsorption can determine the suitability a material for practical application: if the binding is too high, release of the hydrogen will be difficult at moderate operating temperatures, while if the binding is too weak, storage of the hydrogen will be ineffective.

Calculating the energetics of hydrogen adsorption is a difficult task and requires highly accurate quantum mechanics based calculations. If the structure and eventually the dynamics of the adsorption process are to be accurately modeled, the potential energy surface of the adsorbant and adsorbate must be accurately simulated over a length scale of at least 5 Angstrom. Density functional theory (DFT) based methods are the most widely applied electronic structure methods for studies of hydrogen storage materials. However, in practice the DFTs are not only approximate, but are also rarely benchmarked in the non-bonding and weakly bonding configurations vital for hydrogen storage.

Motivated in part by recent discussions and discrepancies for DFT predictions of hydrogen adsorption on alkaline earth metals [1-3], we have performed extensive Quantum Monte Carlo (QMC) [4] calculations for $\mathrm{H}_{2}$ (dihydrogen) adsorption on the $\mathrm{Ca}^{1+}$ system. The ion's charge models the scenario where the ion is absorbed on graphene [5, 6]. QMC provides an accurate and unbiased reference to compare against approximate but more computationally affordable approaches. We concentrate on the interaction of hydrogens with a single ion, as opposed to a system with a substrate, since the geometries are easily and unambiguously specified and the systems are already sufficient to demonstrate substantial differences in predicted binding energies and overall shape of the binding curves.
The interaction of one or many $\mathrm{H}_{2}$ molecules with $\mathrm{Ca}^{1+}$ will undoubtedly involve several effects: charge transfer, polarization, and potential long range dispersion (or van der Waals) interactions. To accurately model these systems, first principles calculations should be able to accurately account for all these effects with little reliance on, e.g. error cancelation. For example, van der Waals interactions interactions are naturally and accurately included within QMC approaches 7 10], but are absent from common DFTs.

In the following we (i) describe our QMC methodology, (ii) present benchmark results for the cases of single $\mathrm{H}_{2}$ adsorption on $\mathrm{Ca}^{1+}$, and (iii) since in actual use scenarios additional hydrogen molecules will be present we also results for quadruple $\mathrm{H}_{2}$ adsorption. These systems are constructed identically to those of Ref. [1]. Finally, (iv) we summarize our findings.

Quantum Monte Carlo - The QMC method allows for a very efficient and accurate solution of the Schrödinger equation. In contrast with many electronic structure methods, QMC methods involve only well controlled approximations. Although their computational prefactor is often large, for small and medium sized molecular systems energetics close to chemical accuracy can be obtained, e.g. Refs. [11, 12]. These properties make QMC methods ideal for benchmark studies and for the assessment of computationally cheaper but more approximate methods.

QMC methods are wavefunction based and the most important input is the trial many body wavefunction. In Variational Monte Carlo (VMC) a direct variational evaluation of the energy of a trial wavefunction is performed using importance sampled Monte Carlo integration. VMC calculations therefore suffer from a potentially very strong dependence on the input wave function and any prior assumptions about the electronic structure, but have the advantage that the actual many-body wavefunction is obtained and can be analyzed. In fixed-node diffusion quantum Monte Carlo (DMC), the lowest energy state consistent with the zeros (nodes) of the trial 
wavefunction is projected. This projection greatly reduces the dependence of the final energy on the input trial wavefunction compared to VMC. In practice, very accurate results are obtained by DMC for a wide variety of molecular and solid state systems [4, 7-10, 13, 14]. Due to the increased robustness we concentrate on DMC results in this study.

For our purposes, the only significant approximations in DMC calculations are (i) the use of pseudopotentials and (ii) the fixed-node approximation and consequent dependence on the nodal surface of the input trial wavefunction. The first approximation introduces systematic errors via the approximate treatment of core-valence interactions and via the locality approximation 15] necessary to evaluate the non-local pseudopotentials in DMC. We minimize these errors by using a small Ne core for the Ca pseudopotential [16] and very high quality trial wavefunctions. We use the same pseudopotentials in all our calculations to ensure a fair comparison between all methods: the same Hamiltonian is solved in our QMC, DFT, and quantum chemical calculations.

To minimize the nodal errors in our DMC calculations we also use multideterminant trial wavefunctions obtained from configuration interaction calculations that are subsequently reoptimized via the energy minimization method[11]. This approach is a significant advance over conventional applications of DMC where the nodal surface of the trial wavefunction consists of only a single Slater determinant determined by a less accurate theory such as DFT: the nodal errors are systematically reduced to near chemical accuracy [11, 12] when sufficient statistics can be obtained and the multideterminant expansion is large enough. Previous studies have shown that (i) for light molecules single determinant DMC yields results similar in accuracy $\operatorname{CCSD}(\mathrm{T})$ with the aug-cc-pVQZ basis set[13], (ii) these errors are further reduced with multideterminant methods, e.g. [11-13], and (iii) pseudopotential errors are small and less significant than the nodal error in these systems [13, 14].

In principle, modern trial wavefunction optimization methods [11, 12] can produce DMC results nearly independent of the input provided sufficiently flexible trial wavefunction forms are adopted. Here we validate our single determinant nodal surface results using large configuration interaction expansions of many determinants.

In the following calculations we use trial wavefunctions consisting of a weighted sum of Slater determinants multiplied by a two-body Jastrow factor. The Slater determinants consist of orbitals determined by GAMESS[17] DFT or complete active space self consistent field (CASSCF) calculations expanded in the large ANO-VTZ gaussian basis set[16]. The two-body Jastrow factor does not change the nodal surface but acts to enforce the electron-electron cusp condition, greatly improving the overall quality of the trial wavefunctions. For the QMC calculations we used the QWALK code 18]. Multideterminant QMC calculations used up to 370 determinants, where we took all CASSCF determinant of squared magnitude greater than 0.01. Energy minimization was performed starting from the truncated CASSCF results. We note that the
DMC energies always lie substantially below the pure quantum chemistry results. Pseudopotentials were derived in the soft Hartree-Fock formalism [16]. An average DMC population of $\sim 30000$ walkers and a small time step of 0.005 a.u. was used. The largest DMC calculations used $\mathrm{O}(1000)$ processor hours per energy point.

Results for single $\mathrm{H}_{2}$ absorption - Figure 1 shows our calculated binding energy curve for the hydrogen dimer on $\mathrm{Ca}^{1+}$, with the molecular bond oriented perpendicularly to the line of approach. Since the calculation of forces is not well developed in QMC, for each distance from the $\mathrm{Ca}^{1+}$ ion we computed energies for all methods with a fixed bond length of 0.77 Angstrom, corresponding to the value found near binding in quantum chemical calculations [1]. Only small changes in the fully relaxed value are seen over all distances, indicating that the trends in the binding at fixed bond length are representative of the relaxed case. For computational simplicity, we treat the energy of the system at $z=4.6$ Angstrom as representing fully separated unbound system.

Our results show that while the potential energy surface varies quantitatively between the methods, for a single dimer the general trends given are qualitatively similar for many of the methods, with a single minimum. However, unrestricted Hartree-Fock (UHF) and second order Møller-Plesset perturbation theory (MP2) calculations show negligible binding. The DMC data shows a minimum around $z=3.1$ Angstrom, and binding of $\sim 0.025 \mathrm{eV}$.

Comparing the density functional results against the DMC energy curve we find that B3LYP[19] functional gives a relatively good agreement, with minimum at $z=<2.9$ Angstrom. However, the LSDA functional [20] significantly overbinds by at least $0.1 \mathrm{eV}$, while the PBE functional[21] lies midway between the B3LYP and LSDA values. None of the functionals results in false energetic minima in the binding energy curve, however the distance of the minimum energy varies by 0.5 Angstrom over these functionals. Calculations using HartreeFock exchange combined with PBE correlation (HFX+PBEC) (similar to Refs. 22, 23 except with $100 \%$ exchange) very closely resemble the DMC results; analysis and possible reasons for the apparent accuracy are discussed after the four dihydrogen results.

Results for four $\mathrm{H}_{2}$ absorption - Figure2 2 shows our calculated binding energy curve for four hydrogen dimers on $\mathrm{Ca}^{1+}$. In this system the hydrogens are pinned in a planar geometry, ninety degrees apart in $D_{4}$ symmetry, with molecular bonds oriented perpendicularly to the line of approach (inset in Figure 2).

The binding energies obtained with single determinant DMC display a minimum around 2.2 Angstrom. However, comparing these energies with those over 3 Angstrom clearly shows the minimum to be a local metastable minimum: there is no binding of four $\mathrm{H}_{2}$ molecules in this planar geometry at short range at the single determinant DMC level. To test the accuracy of these calculations we also used multideterminant wavefunctions determinants, initially obtained by restricted active space RAS $(9,37)$ calculations. The binding is 




FIG. 1: Calculated binding energy for a single hydrogen molecule $\left(\mathrm{H}_{2}\right)$ approaching $\mathrm{Ca}^{1+}$. The molecule to oriented with the bond perpendicular to the line of approach (see inset). Results are shown for the unrestricted Hartree-Fock (UHF), the local spin density approximation (LSDA), PBE and B3LYP density functionals, second order Møller-Plesset perturbation theory (MP2). We also show Diffusion Quantum Monte Carlo (DMC) and density functional results calculated using exact exchange combined with PBE correlation (HFX+PBEC). The DMC calculations use a single determinant of B3LYP orbitals and a Jastrow factor for the trial wavefunction and nodal surface. The hydrogen molecule bond length is held fixed at 0.77 Angstrom. The lines are a guide to the eye. Error bars are smaller than the size of the DMC symbols.

shifted to higher energies by $\sim 0.1 \mathrm{eV}$ indicating that the single determinant results and nodal surface are robust.

DFT based calculations show clear energy minima around the 2.2 Angstrom distance indicating significant binding of the $\mathrm{H}_{2}$ molecules. Although the depth of binding varies, similar behavior is obtained for LSDA, PBE and B3LYP. The same energetic ordering is observed as for the single hydrogen case, with LSDA displaying greatest binding. By contrast, UHF calculations display only a slight minimum around 2.3 Angstrom. We also include quantum chemical results from RAS and complete active space (CAS) calculations in Fig 2 The larger active space calculations reduce the calculated binding energy, moving the quantum chemical results towards the DMC results. Perturbative theory results (MP2) also show no overall binding. A clear transition between states of $A_{1 g}$ and $B_{2 g}$ symmetry is observed[1] between 2.5 and 3.0 Angstrom depending on the underlying theory.

Our qualitative conclusion of no binding for the four hydrogen molecule case with fixed 0.77 Angstrom bond length is in qualitative agreement with previous quantum chemical results[1]. However our more extensive basis sets and more rigorous DMC calculations reveal that that the energy scale for any potential binding is very small, only a few tenths of an $\mathrm{eV}$. Strikingly, even allowing a generous estimate of $0.2 \mathrm{eV}$ residual systematic errors in our DMC calculations, any eventual binding will remain small (order $0.1 \mathrm{eV}$ ) whereas the LSDA and PBE functionals predict binding energies one order of magnitude larger.

We also tested energies obtained from density functional theory using using Hartree-Fock exchange combined with PBE correlation, gradually increasing the fraction of exchange. As for the single hydrogen molecule case, the calculated binding curve accurately follows the DMC data over all distances. However, only a $100 \%$ contribution fully reproduced the DMC data; lesser contributions smoothly interpolating between the DMC and PBE results. Given the wide variation seen for other functionals, this is noteworthy, and also indicates the primary source of error in the other density functional predictions: We argue, in accordance with Ref. [24], that the semi-local functionals provide good description of static correlation and exchange in this system. On the other hand, dynamic or long-range exchange is mostly absent in these functionals. Since our system seems not to have a strong multi-reference character (based on our RAS and CAS calculations) the dynamical part of exchange must play a dominant role. This last part is well described only in HF or in exact-exchange functionals such as optimized effective potential (OEP) method. Therefore including full dynamical part of a exchange together with the correlation from LSDA, PBE, or B3LYP gives a good description. This observation is mostly independent from the type of the correlation used.

Summary - We have performed benchmark quantum Monte Carlo calculations of hydrogen molecule binding on the Ca cation. Density functional calculations vary widely in the predicted binding energy. Density functional calculations using Hartree-Fock exchange well reproduce the Monte Carlo results, suggesting a route - if not universal - to predictive and accurate calculations in this and related systems. We hope that our results will help further motivate the development of improved functionals. As metal ions have been proposed as hydrogen binding centers in new hydrogen storage materials, we strongly recommend caution in applying density functional methods to these systems. Appropriate benchmarking using quantum chemical or quantum Monte Carlo techniques is required.

We thank V. R. Cooper for helpful conversations. This research used computer resources supported by the U.S. DOE Office of Science under contract DE-AC02-05CH11231 (NERSC) and DE-AC05-00OR22725 (NCCS). Research sponsored by U.S. DOE BES Division of Materials Sciences \& Engineering (FAR) and ORNL LDRD program (MB). The Center for Nanophase Materials Sciences research was sponsored by the U. S. DOE Division of Scientific User Facilities (PRCK).

[1] J. Cha, S. Lim, C. H. Choi, M. Cha, and N. Park, Physical Review Letters 103, 216102 (2009).

[2] Y. Ohk, Y. Kim, and Y. Jung, Physical Review Letters 104, 179601 (2010).

[3] J. Cha, C. H. Choi, and N. Park, Physical Review Letters 104, 179602 (2010). 


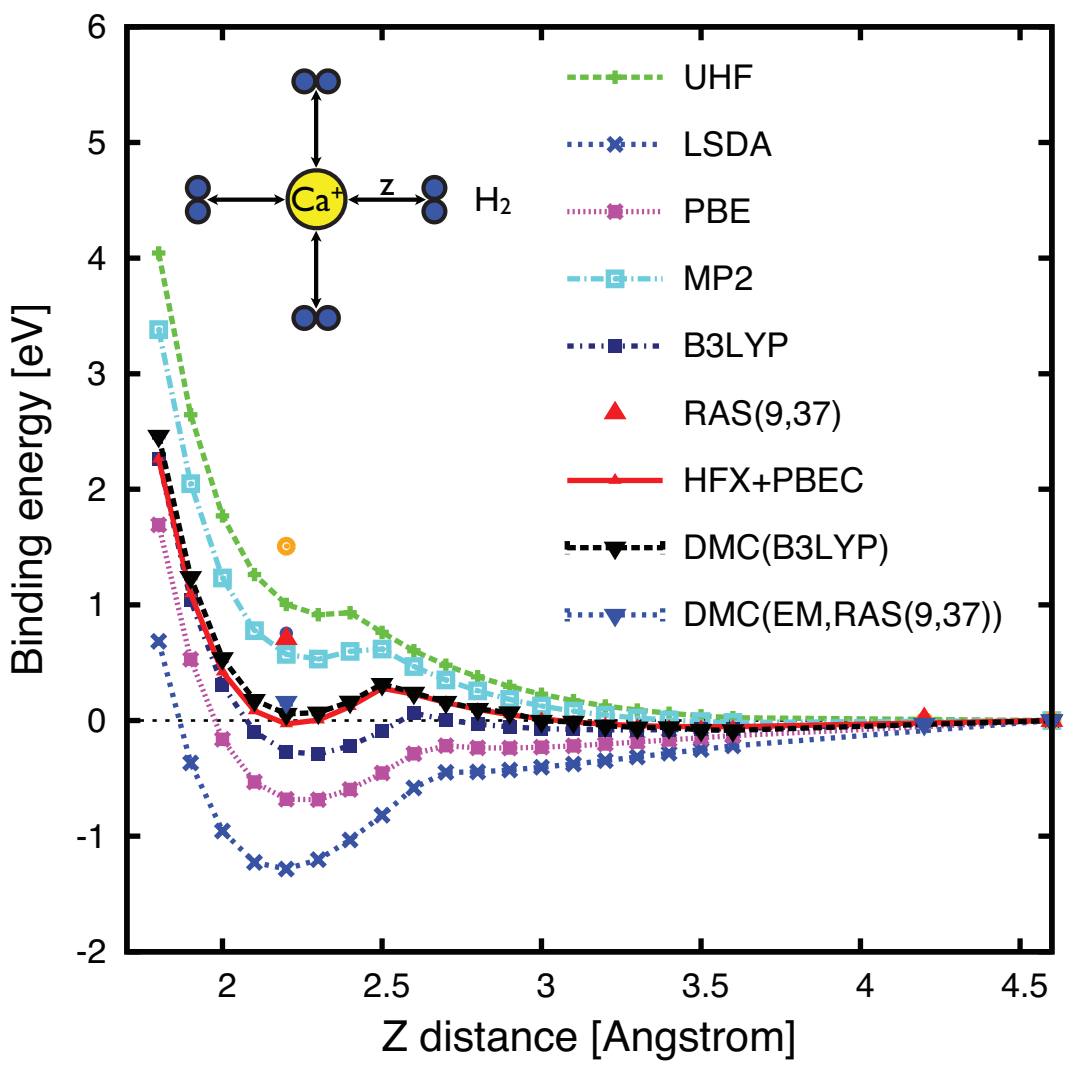

FIG. 2: Calculated binding energy for a four dihydrogen $\left(\mathrm{H}_{2}\right)$ molecules approaching $\mathrm{Ca}^{1+}$. Each molecule is oriented with the bond perpendicular to the line of approach in an overall planar geometry with D4 symmetry (see inset). Results are shown for unrestricted Hartree-Fock (HF), density functional theory using several approximate functionals (LDA, PBE, B3LYP), and for two sets of Diffusion Quantum Monte Carlo (DMC) calculations. For the range of distances studied, DMC data is given for a single determinant of B3LYP orbitals and a Jastrow factor for the trial wavefunction and nodal surface (black triangles). At 2.2 Angstrom separation we also compute the binding energy in DMC using Energy Minimized Restricted Active Space (RAS) multideterminant wavefunctions. At this distance we also show quantum chemical results for RAS $(9,37)$ (red triangle), RAS $(9,31)$ (blue circle), and complete active space CAS $(9,18)$ (orange circle). HFX+PBEC indicates density functional results calculated using exact exchange combined with PBE correlation. The hydrogen molecule bond lengths are held fixed at 0.77 Angstrom. See text for more information. The lines drawn are a guide to the eye. DMC error bars are smaller than the symbols.

[4] W. M. C. Foulkes, L. Mitas, R. J. Needs, and G. Rajagopal, Reviews of Modern Physics 73, 33 (2001).

[5] C. Ataca, E. Aktrk, and S. Ciraci, Physical Review B 79, 041406 (2009).

[6] G. Kim, S. Jhi, S. Lim, and N. Park, Physical Review B 79, 155437 (2009).

[7] S. Sorella, M. Casula, and D. Rocca, Journal of Chemical Physics 127, 014105 (2007).

[8] T. D. Beaudet, M. Casula, J. Kim, S. Sorella, and R. M. Martin, Journal of Chemical Physics 129, 164711 (2008).

[9] B. Santra, A. Michaelides, M. Fuchs, A. Tkatchenko, C. Filippi, and M. Scheffler, Journal of Chemical Physics 129, 194111 (2008).

[10] J. Ma, D. Alfe, A. Michaelides, and E. Wang, Journal of Chemical Physics 130, 154303 (2009).

[11] C. J. Umrigar, J. Toulouse, C. Filippi, S. Sorella, and R. G. Hennig, Physical Review Letters 98, 110201 (2007).

[12] M. Bajdich, M. L. Tiago, R. Q. Hood, P. R. C. Kent, and F. A. Reboredo, Physical Review Letters 104, 193001 (2010).

[13] J. C. Grossman, Journal of Chemical Physics 117, 1434 (2002).

[14] N. Nemec, M. D. Towler, and R. J. Needs, Journal of Chemical Physics 132, 034111 (2010).
[15] L. Mit, E. L. Shirley, and D. M. Ceperley, Journal of Chemical Physics 95, 3467 (1991).

[16] M. Burkatzki, C. Filippi, and M. Dolg, Journal of Chemical Physics 126, 234105 (2007).

[17] GAMESS Version 12 JAN 2009 (R1) From Iowa State University. M.W. Schmidt, K.K. Baldridge, J.A. Boatz, S.T. Elbert, M.S. Gordon, J.H. Jensen, S. Koseki, N. Matsunaga, K.A. Nguyen, S.J. Su, T.L. Windus, M. Dupuis, J.A. Montgomery, J. Comput. Chem. 14, 1347-1363 (1993).

[18] L. K. Wagner, M. Bajdich, and L. Mitas, Journal of Computational Physics 228, 3390 (2009).

[19] A. D. Becke, Physical Review A 38, 3098 (1988).

[20] J. P. Perdew and A. Zunger, Physical Review B 23, 5048 (1981).

[21] J. P. Perdew, K. Burke, and M. Ernzerhof, Physical Review Letters 77, 3865 (1996).

[22] M. Ernzerhof and G. E. Scuseria, Journal of Chemical Physics 110, 5029 (1999).

[23] C. Adamo and V. Barone, Journal of Chemical Physics 110, 6158 (1999).

[24] S. Kummel and L. Kronik, Reviews of Modern Physics 80, 3 (2008). 